constructs. A new mathematical approach (Rasch analysis) then allows for formal tests of unidimensionality in ordinal scales. Questions, or tasks which are summated in some fashion to give an overall score must be unidimensional. Furthermore, at the same level of ability (for example in walking), the probability of a patient doing a task must be the same irrespective of whether they are male or female, young or old, underlying pathology (cross-diagnostic validity) or country of use (cross-cultural validity) - the absence of DIF.

Once these fundamental requirements of measurement are met, tests of reliability, validity and responsiveness may proceed. In this way, those working in the field of arthritis can be confident that evaluation of interventions are supported by high quality measurement.

\section{SP0059 CROSS-CULTURAL VALIDATION OF PERCEIVED HEALTH STATUS MEASURES}

F Guillemin. School of Public Health, Faculty of Medicine, Vandoeuvre-Les-Nancy, France

\subsection{6/annrheumdis-2001.16}

Since difference in country, language or culture make people vary in disease expression, in perception of health problems and quality of life, and in their use of health care systems, there is a need for measures tailored to their particular need. It is also increasingly necessary to have standardised instruments for international use in research and practice. To resolve this paradox, the process of cross-cultural validation will particularly focus on the achievement of equivalence in concept measured across culture.

Perceived health status measures are complex scales in the form of interview or self-report questionnaires. Their validation shall follow the standards of psychometric validity (content, construct, reliability and sensitivity to change). The content validity is particularly challenging the cross-cultural validation. Among options to conduct validation of the content, it is possible to achieve it by:

- building an instrument concomitantly in several countries. It allows to work together in the generation and selection of items, as has been done with disease-specific (QUALEFFO, Womac), or generic instruments (WHOQOL).

- developing a standard methodology to cross-culturally adapt an established instrument offering guarantee of equivalence between source and target. The participation of the developers to the process is highly contributive to clarify concept explored in the original language

Researchers are most frequently facing the situation where the original version has been developed and validated in a foreign language. Thus the second option will be particularly useful.

Simple translation/back-translation is far from meeting basic requirements for equivalence in content. Perfect bilingual experts are scarce, if any.

Guidelines are proposed to ensure cross-cultural content equivalence. They include:

- several translations, with appropriate translators

- expert committee review, including various experts (health professionals, patients) and having specified tasks

- several back-translations, with standardised rules

- testing with patients to probe understandability
The place and role of back-translation has been debated, since its contribution to the process may be overestimated. It is as fallible as translations, and not any (back) translator would be capable of assessing proximity between versions.

From qualitative research, it is now clear that a thoughtful committee working on several translations achieved by adequate translators may well achieve the goal.

One aspect of the cross-cultural adaptation is the potential bias introduced when adapting answer modalities. The scalability of the adapted instrument can be assessed using the Rasch modelling statistic.

Other aspects of validation of the cross-culturally adapted measure should be clearly considered separately. As far as the population in the target culture may differ in many aspects compared to the original targeted population, especially in many characteristics relating to culture, a standard procedure of validation should be conducted:

- construct validity will consider how items and dimensions are clearly exploring separate and complementary facets of the concept, i.e. health status, and the coherence of scales (factor structure and internal consistency)

- convergent validity will be documented by the relation to variables exploring a similar construct,

- discriminant validity will be documented by checking how groups with known difference will be recognised as different by the instrument

- reliability will be documented by the ability of the measure to produce the same value when exploring the same phenomenon

- sensitivity to change will be its power to detect intra individual changes over time.

These steps are mandatory requirements to document the equivalence of the measurement, at the same degree of reliability in different cultural settings. Because it is much shorter process than developing a new instrument in each and every country, and since many instruments are already available, the cross-cultural adaptation and validation of existing perceived health status and quality of life measures is highly recommended.

\section{State of the art lecture - Friday 15 June, 10.00-10.30/Congress Hall}

\section{SP0060 DISEASE MODIFYING DRUGS IN THE TREATMENT OF RHEUMATOID ARTHRITIS}

M Weinblatt. Rheumatology, Brigham and Women's Hospital, Boston, USA

10.1136/annrheumdis-2001.17

The last several years have been very exciting for the therapy of rheumatoid arthritis (RA). One major advance has been the acceptance of a core set of disease activity measurements and definition of improvement. The American College of Rheumatology (ACR) core set of disease measurements includes tender and swollen joint count, physician and patient assessment of disease activity, patient assessment of pain, physical function and acute phase reactants (Arthritis Rheum. 36:729,1993). The ACR definition of response requires a $20 \%$ improvement in both tender and swollen joint count and a 20\% improvement in three of the five remaining ACR core set measures (Arthritis Rheum.38:727,1995). Another disease activity response rate developed by Dutch investigators is called the DAS (Disease 